\title{
Proper Orthogonal Decomposition and Statistical Analysis of 2D Confined Impinging Jets Chaotic Flow
}

DOI:

10.1002/ceat.201900050

\section{Document Version}

Accepted author manuscript

Link to publication record in Manchester Research Explorer

\section{Citation for published version (APA):}

Torres, P., Goncalves, N. D., Fonte, C. P., Dias, M. M., Lopes, J. C. B., Liné, A., \& Santos, R. J. (2019). Proper Orthogonal Decomposition and Statistical Analysis of 2D Confined Impinging Jets Chaotic Flow. Chemical Engineering and Technology. https://doi.org/10.1002/ceat.201900050

\section{Published in:}

Chemical Engineering and Technology

\section{Citing this paper}

Please note that where the full-text provided on Manchester Research Explorer is the Author Accepted Manuscript or Proof version this may differ from the final Published version. If citing, it is advised that you check and use the publisher's definitive version.

\section{General rights}

Copyright and moral rights for the publications made accessible in the Research Explorer are retained by the authors and/or other copyright owners and it is a condition of accessing publications that users recognise and abide by the legal requirements associated with these rights.

\section{Takedown policy}

If you believe that this document breaches copyright please refer to the University of Manchester's Takedown Procedures [http://man.ac.uk/04Y6Bo] or contact uml.scholarlycommunications@manchester.ac.uk providing relevant details, so we can investigate your claim.

\section{OPEN ACCESS}




\author{
Pedro Torres ${ }^{1,2, *}$ \\ Nelson D. Goncalves ${ }^{1}$ \\ Claudio P. Fonte ${ }^{2}$ \\ Madalena M. Dias ${ }^{1}$ \\ José Carlos B. Lopes ${ }^{1}$ \\ Alain Liné ${ }^{3}$
}

Ricardo J. Santos ${ }^{1, \dagger}$

\title{
Proper Orthogonal Decomposition and Statistical Analysis of 2D Confined Impinging Jets Chaotic Flow
}

The Proper Orthogonal Decomposition (POD) is shown to be a statistical operation that identifies the main characteristics of chaotic flows and separates them into few modes. The dynamic chaotic flow is obtained from 2D Computational Fluid Dynamics (CFD) simulations, for different Reynolds numbers, of a Confined Impinging Jets mixer (CIJ). The POD enables a reconstruction of the dynamic flow from a few modes that are related with coherent flow structures. The POD flow reconstruction enables a large compression of the flow data set. The decomposition of the flow field in orthogonal modes related to coherent structures, provides a direct insight into the mixing dynamics and scales, which are not accessible from flow dynamics statistic quantities that were introduced in the context of turbulence and are here applied to a chaotic flow.

Keywords: CFD, Chaotic Flows, CIJ, POD.

\author{
Author affiliations \\ ${ }^{1}$ Laboratory of Separation and Reaction Engineering, Associate Laboratory LSRE-LCM, \\ Department of Chemical Engineering, Faculdade de Engenharia, Universidade do Porto, Rua \\ Dr. Roberto Frias, 4200-465, Porto, Portugal. \\ ${ }^{2}$ The University of Manchester, School of Chemical Engineering \& Analytical Science, Oxford \\ Road, ManchesterM13 9PL, UK. \\ ${ }^{3}$ Université de Toulouse, LISBP, INSA, INRA, CNRS, Toulouse, France. \\ Email corresponding author: * pedro.torres@manchester.ac.uk, ${ }^{\dagger}$ rsantos@fe.up.pt
}

\section{Introduction}

Proper Orthogonal Decomposition (POD) is a tool that extracts small dimensional data from complex high dimensional processes ${ }^{[1]}$. This procedure is used, in the context of turbulence and chaotic flows, to obtain dominant features and trends - like coherent structures - in space and time ${ }^{[2]}$. One of the first uses of POD in turbulence was by Lumley in 1967, but according to Holmes, other uses of either POD or similar Reduced Order Methods have been made earlier in other fields of work by Kosambi ${ }^{[3]}$, Loève ${ }^{[4]}$ and Karhunen ${ }^{[5]}$. The Kosambi-Karhunen-Loève theorem ${ }^{[6]}$, or simply the Karhunen-Loève theorem, was extended as a way of applying the POD to infinite-dimensional spaces, as it uses single parameter functions instead of vectors ${ }^{[1]}$.

The POD capability to extract coherent structures, which exhibit patterns in space and also in time, is particularly relevant in mixers, because these structures are the basis of the mixing 
mechanisms: folding and stretching of fluid elements ${ }^{[7,8]}$. POD has been applied to chemical engineering in the analysis of stirred tanks ${ }^{[9-12]}$ and recently orbitally shaken reactors ${ }^{[13]}$.

POD decomposes complex data into sets of modes, which in the context of turbulent flows, enables to extract the oscillatory motions present in the flow and separate them not only by associated kinetic energy but also by wave number. In the present work, the modes are represented by vector maps. Another use of POD is the possibility of reconstructing the flow using only some of the modes (i.e. the dominant features and trends of the flow), providing a significant data compression that can also facilitate new calculations of flow dependent properties (such as mass transfer) from the reduced data set reconstruction, without requiring a new set of experiments or numerical calculations.

A mixing device that explores complex flow patterns for fast mixing of fluids is the confined impinging jets $(\mathrm{ClJ})$ mixer ${ }^{[14-18]}$ which is based on the principle that the energy dissipated from two impinging jets promotes mixing rapidly and without movable mechanical parts ${ }^{[19]}$. The flow dynamics in ClJs were proven to be mainly $2 \mathrm{D}$ in nature ${ }^{[20]}$ and so $2 \mathrm{D}$ CFD simulations are often used to study these mixers.

POD is applied to the flow in CIJs aiming to extract average and coherent flow structures, which enable a better insight into a mixer that has been thoroughly studied from dynamic analysis tools and mixing simulations ${ }^{[20,21]}$. The paper also aims to explore the range of application of POD in chaotic flows, two particular cases are analyzed: the interpretation of POD of a chaotic flow and comparison of this decomposition with the computation of flow integral length scales; and the application of POD for flow data compression, which enables the usage of large data sets on post-CFD simulation mixing studies of the CIJs, for example with Lagrangian methods ${ }^{[22]}$.

\section{Case Study}

The CFD model for the 2D ClJs used in this work was previously validated from extensive numerical and experimental studies ${ }^{[14-18]}$. The geometry of the ClJs is show in Fig. 1 and consists of a $2 \mathrm{D}$ rectangular chamber with two opposed injectors near the top of the chamber, and an outlet in the bottom of the chamber. The chamber width, $D$, is $1 \times 10^{-2} \mathrm{~m}$, the inlet channels width, $d$, is $1.5 \times 10^{-3} \mathrm{~m}$ and the length of the chamber, $L$, is $5 \times 10^{-2} \mathrm{~m}$. The $x$ and $y$ coordinates start at the impact point of the opposed jets, which is situated where the axis of the inlet channels crosses the axis of the chamber.

The simulations were done using the numerical integration of the Navier-Stokes and conservation of mass equations, via the software ANSYS Fluent ${ }^{\circledR}$. The Navier-Stokes equation was used in this work with the following form for the momentum conservation,

$\rho \frac{D \boldsymbol{u}}{D t}=-\nabla p+\mu \nabla^{2} \boldsymbol{u}$

where $\rho$ is the density, $\boldsymbol{u}$ is the velocity vector, $t$ is the time, $p$ is the pressure and $\mu$ is the viscosity.

The conservation of mass equation, for an incompressible flow, is expressed as

$\nabla \cdot \boldsymbol{u}=0$

The chamber and channels walls have a no-slip condition $(\boldsymbol{u}=0)$ throughout the simulation.

The inlet $y$-velocity profile normal to the injectors is defined as

$u_{y}(x)=1.5 v_{i n j}\left(1-\left(\frac{x}{d / 2}\right)^{2}\right)$

where $v_{i n j}$ is the average velocity at the inlets. The sign of the inlet velocity is positive at the bottom inlet and negative at the opposite side. The outlet has a gauge pressure of 0 . The simulations were made for Reynolds number, $\mathrm{Re}$, in a range from $\mathrm{Re}=250$ to 500 , and the average velocity at the inlets is given by the Reynolds number defined as 
In these simulations, the fluid properties used were $\rho=1000 \mathrm{~kg} / \mathrm{m}^{3}$ and $\mu=0.03 \mathrm{~Pa} \cdot \mathrm{s}$ and the cell size defined for the mesh is $62.5 \mu \mathrm{m}$. The time-step size is calculated from

$\Delta t=\frac{2}{3} \frac{\Delta x}{v_{\text {inj }}}$

in which $\Delta t$ is the time-step and $\Delta x$ is the size of each cell. The inlet velocity and time-step size for each Reynolds number is shown in Tab. 1.

A steady state flow solution was calculated initially. The pressure-velocity coupling was done by using the Coupled scheme. The pressure gradient terms were discretized using the PRESTO! scheme, and the Second Order Upwind scheme was used to discretize the advective terms of Eq. 1.

From that initial flow solution, a dynamic flow simulation was started, extending through 5 times the residence time of the CIJs $\left(\tau=(D H) /\left(2 v_{i n j} d\right)\right)$, and the results were only recorded for POD analysis in the following 5 residence times $(5 \tau)$. This procedure aims at eliminating the influence of the starting conditions where the flow evolves to a fully developed chaotic flow. The analysis of this transient period was made by Santos et al. ${ }^{[14]}$, which demonstrated that the evolution from steady state to a fully developed chaotic flows extends for approximately $2 \tau$. Afterwards, the dynamic flow has no evolution of the dynamical behaviour averaged features.

The velocity vector field of the flow in the ClJs at the instant $t=5 \tau$, for $\operatorname{Re}=300$, is shown in Fig. 2. The flow is mainly determined by two jets facing each other in the top of the chamber $(x=$ $\left.5 \times 10^{-3} \mathrm{~m}\right)$. In the top of the mixing chamber $\left(0 \leq x \leq 5 \times 10^{-3} \mathrm{~m}\right)$ there is a headspace where two vortices are formed, one in each side of the chamber. The jets impingement point is slightly downstream of the impact point $\left((x, y)=\left(5 \times 10^{-3} \mathrm{~m}, 0 \mathrm{~m}\right)\right)$, due to the bending of the jets before impinging on each other. Downstream from the jets' impingement, close to the mixing chamber axis, two small vortices are created at each side of the jets. These vortices engulf to create a larger vortex with diameter $D$ at approximately $x=3 D / 2$. These larger vortices form a vortex street flowing towards the mixing chamber outlet.

\section{$3 \quad$ Results}

\subsection{Turbulence Integral Lengthscales}

The turbulence integral scale, $L_{11}$, is obtained from classical methods of turbulence, it is based on correlating dynamic data at different distances. This scale is going to be used for comparison with POD results. The $L_{11}$ is computed from the values of the $y$ component of the velocity $\left(u_{y}\right)$ in a line throughout the chamber axis from the jet impact point $(x=0)$ to the outlet for the recorded data set with $5 \tau$. Only the values of $u_{y}$ were used because these fluctuations are the ones that most clearly show the evolution of the vortices through the mixing chamber. The scales are calculated from the time average of the autocorrelation of the velocity values throughout a line, in this case the axis of the mixing chamber, defined as

$L(x, r)=\frac{\langle u(x+r, t) u(x, t)\rangle}{\langle u(x, t) u(x, t)\rangle}$

where $r$ is positive and represents the displacement between the two points. The lengthscales are calculated by the integral between the zero scale, $r=0$, where the normalized autocorrelation is 1 , and the point where this autocorrelation becomes zero, this is $L_{11}(x)=\int_{r=0}^{r \mid L=0} L(x, r) d r$. The $L_{11}$ is a characteristic lengthscale of the larger eddies ${ }^{[23]}$. These scales evolution throughout the mixing chamber are shown in Fig. 3.

The turbulent integral lengthscale of this flow is on average close to $0.85 D$ for $x>2 D$, meaning that the eddies formed are almost the size of the mixing chamber and so their size is restricted 
by the mixer walls. It can also be noticed that the vortices at around $2.5 \mathrm{~mm}$, which is close to the contact point of the jets, are about half the width of the chamber, evolving to larger sizes as they progress towards the outlet. These turbulent lengthscales evolution is verified by the vector field seen previously in Fig. 2, where at the impingement point there are two vortices with a diameter close to half the width of the chamber. These eddies engulf and evolve into larger eddies near the outlet, which is demonstrated with the increase of lengthscale near the outlet.

The turbulent lengthscale is not computed over the entire axis of the mixing chamber, because the space left between the last points shown in Fig. 3 and the outlet is too short for a complete eddy, hence making it impossible to have correlation values equal to zero, $L(x, r)=0$.

\subsection{Proper Orthogonal Decomposition}

The Proper Orthogonal Decomposition of the ClJs flow, in this case the $x$ and $y$ components of velocity at all cell centres of the mesh, is applied to the dynamic datasets comprising $5 \tau$ that were recorded from the CFD simulation. The data was analysed with a script made in MATLAB, which organized the data as a matrix $\boldsymbol{X}$ where at each $i$ row is the entire collection of time steps at a single point for a component of velocity (either $u_{y}$ or $\left.u_{x}\right)$, thus $X(i, j)=u_{x}\left(x_{i}, y_{i}, t_{j}\right)$ and $X(N+$ $i, j)=u_{y}\left(x_{i}, y_{i}, t_{j}\right)$, where $u_{x}$ and $u_{y}$ represent the velocity in the $x$ and $y$ components, respectively. The first $N$ points of the $\boldsymbol{X}$ matrix represent the values of $u_{x}$ for all points of the mesh and then the bottom half of the matrix from $N+1$ to $2 N$ represent the same for $u_{y}$.

To apply POD, one needs to calculate the autocovariance matrix of $\boldsymbol{X}$, as

$\boldsymbol{M}=\boldsymbol{X} \boldsymbol{X}^{T}$

The eigenvalues $(\lambda)$ and eigenvectors $(\boldsymbol{\alpha})$ of the autocovariance matrix $\boldsymbol{M}$ are the results of the POD. The eigenvalues for each Re are organized in a spectrum, according to their relative magnitude (Fig. 4), and the sum of all eigenvalues is the total kinetic energy of the flow ${ }^{[24]}$. In addition to the representation used in Fig. 4, these spectra can also be represented using cumulative eigenvalues. The eigenvalues stand for the energy of each mode, being that lower modes contain more energy. The modes are obtained from the eigenvectors, which have the same amount of points as the original mesh. Thus, from the eigenvectors of matrix $\boldsymbol{M}$ a vector map is reconstructed using the same coordinates of the original vector field. These vector fields from the eigenvectors, for $\mathrm{Re}=300$, are shown in Fig. 5 .

It is clear from the eigenvalue spectra in Fig. 4 that the first mode is the most important one, as it is the one carrying the most kinetic energy. The 4 first modes are shown in Fig. 5 as vector maps. The first mode depicts the average flow field. The average flow field is characterized by the impingement of the opposed jets at the mixing chamber axis and an increase of the jets overall flow section when they are bent towards the outlet. The increase on the averaged flow section of the jets is due to the dispersion of the chaotic flow paths as can be seen from the dynamic flow field in Fig. 2. From dynamic simulations the jets sections are observed to be kept almost unchanged from inlet to outlet, although the jets path is extended to flow around the vortices ${ }^{[16]}$.

The second and third mode represent the larger eddies, which are the ones carrying most energy in the flow and are responsible for the main mixing mechanisms. These eddies are orthogonally placed i.e. they are exactly displaced by $\pi / 2$ as can be seen from the fact that in the eigenvector vector map for Mode 2 and 3 in Fig. 5 the vortices centres are completely out-of-phase. So, the POD modes are pairs of orthogonal modes and thus can be linearly combined to make the vortices evolve throughout the mixing chamber. Mode 2 and 3 have the same energy and are clearly the two most important modes after Mode 1 (Fig. 4).

The flow has some periodicity, but it is not oscillatory. In actuality, it has been clearly shown that this flow is chaotic, in both Lagrangian and Eulerian sense ${ }^{[16]}$. So, the vortices do not evolve in fully repeatable, completely predictive pattern, but have some complex dynamics associated to them that transfers energy to larger modes, which are also required to enable the full description 
of these complex dynamics. The vortices chaotic nature, seen by their irregular shape in Fig. 2 , increases with Reynolds number. Thus, the concentration of energy at higher modes is increasing with the Reynolds numbers, as can be seen in Fig. 4. At lower Reynolds numbers the flow is more regular, though already chaotic, and thus the information is concentrated in the lower modes.

POD enables the turbulence scales that were measured from the $L_{11}$. Noticeably, Mode 2 and 3 show the same scale evolution of the eddies that is observed from $L_{11}(x)$ in Fig. 3: for $0.5 D<$ $x<D$ the vortices diameter is $\approx D / 2$, and further downstream, $2.5 D<x$, the vortices scale increase up to $\approx D$.

\subsubsection{POD coefficients}

To reconstruct the instantaneous velocity field, one must multiply the eigenvectors by a variable regarding time. This variable is the POD coefficient, represented by $a_{k}^{n}$, where $n$ is the number of the mode and $k$ is the time-step. These are calculated from the original velocity field $(\boldsymbol{U})$ at timestep $k$, as such:

$a_{k}^{n}=\boldsymbol{U}_{k} \cdot \boldsymbol{\alpha}_{\mathrm{n}}$

where $\boldsymbol{\alpha}_{n}$ is the $n^{\text {th }}$ eigenvector. The reconstruction is then made by

$\widetilde{\boldsymbol{U}}_{k}=\sum_{n=1}^{\mathcal{N}} a_{k}^{n} \boldsymbol{\alpha}_{\boldsymbol{n}}$

with $\mathcal{N}$ as the number of modes, and $\widetilde{\boldsymbol{U}}_{k}$ being the reconstructed field at time $k$.

The POD coefficients provide information on how the modes are related to one another. In Fig. 6 it is shown the phase plots of the POD coefficients for modes 2 and 3 , showing that when plotted against each other, they form a circular shape, indicating that there is an orthogonal phase shift between them. This sort of behaviour is mostly evident for the lower Reynolds, where there is a greater line density in the circular area of the relation. For larger Reynolds number there is an increase of the chaotic behaviour of the flow, which is seen from larger deviation of the phase plot from the circular shape.

\subsubsection{Flow reconstruction}

According to Sirovich ${ }^{[25]}$, the number of modes for reconstruction should be such that the captured energy (the magnitude of the eigenvalues) in them is at least $90 \%$ of the total kinetic energy and that no neglected mode contains more than $1 \%$ of the total energy. According to this, to have a reconstruction that would capture the required kinetic energy one would need 23 modes. The reconstructions from 5 modes, 10 modes and 100 modes are shown in Fig. 7, along with the representation of the original flow field for comparison.

As the number of modes increases, the actual shape of the vortices and their deformation dynamics becomes also increasingly more apparent. This is the focus of the POD: with only 5 modes one can detect the largest flow structures formed in the flow, but in the particular case of CIJs, these coherent structures have some degree of deformation due to the chaotic nature of this flow ${ }^{[16]}$. Information from these deformations lies in higher modes of lesser energy and is still important for the actual flow reconstruction.

To compare the reconstruction in a more precise manner, the velocity magnitude plot on a single point is displayed in Fig. 8 . The point chosen is the impact point of the jets $(x=D / 2, y=D / 2)$, as it is one of the points with the most extreme dynamics in ClJ.

In this representation of the various reconstructions, it is possible to verify that an increase in the number of modes used translated into a more accurate reconstruction of the flow dynamics. Although the reconstruction with 10 modes proved better than the one with 5 modes (the latter only describes an overall trend whereas the former does have some peaks corresponding to the peaks in the original data), only by using 100 modes there is an accurate description of the original data. The POD reconstruction with fewer modes clearly filters the higher frequencies, which is quite clear in the reconstructed dynamic datasets of Fig. 8. 
To add to this comparison, the Pearson's correlation and determination coefficients are calculated. The results from this comparison are shown in Fig. 9. It's noticeable that even with the required 23 modes, both coefficients wouldn't achieve a value over 0.90 , which in this case might indicate that there is still information from flow chaotic dynamics at larger modes. Along with the coefficients calculated, Fig. 9 also shows the data compression factor that is calculated as the ratio between the total number of points in the original data and the number of points used in the reconstruction:

Data Compression Factor $=\frac{N_{y} N_{x} N_{t}}{N_{x} N_{y} \mathcal{N}+N_{t} \mathcal{N}}$

where $N_{x}$ is the size of the dataset in $x$ dimension, $N_{y}$ is the size of the dataset in $y$ dimension, $N_{t}$ is the number of time-steps and $\mathcal{N}$ is the number of modes used in the reconstruction. One can see that, expectedly, the increase in number of modes translated into a decrease of the compression factor. However, although the plot tends to 0 , at $N_{m}=100$ the compression factor is still above 30. This is, POD enables to compress the original dataset by more than an order of magnitude while maintaining all the relevant information. For applications where higher frequencies are not required, compression factors can be several orders of magnitude.

\section{Conclusions}

Confined Impinging Jets mixers are mixing devices where dynamic flow structures generate a thin lamellar structure of two fluids ${ }^{[18]}$. The mixing flow structures are not clear from traditional turbulence statistics, even from the spatial distribution of such variables such as turbulent energy dissipation rate maps and turbulent kinetic energy. Some information can be summarized in the spectral analysis of turbulence or in the computation of turbulent scales. The focus of this paper is the Proper Orthogonal Decomposition of the flow and the analysis of the flow spatial and dynamics information that is obtained from such decomposition. The amount of information from $\mathrm{POD}$ is also analysed regarding the dynamic flow reconstruction.

The turbulence integral scales are calculated and the evolution of the larger eddies average size throughout the $\mathrm{CIJ}$ mixing chamber is clearly obtained. For the $\mathrm{ClJ}$ the coherent structures increase throughout the chamber, being about half the width of the chamber at the contact point of the opposed jets and extending to about $80 \%$ to $90 \%$ of the mixing chamber width downstream the mixing chamber middle.

One can see from the eigenvalue spectra that the most important mode, the $1^{\text {st }}$ mode, is the average flow, followed by the $2^{\text {nd }}$ and $3^{\text {rd }}$ modes, which contain information of the large eddies' evolution in the mixing chamber. The contribution of larger modes, such as the $4^{\text {th }}$ and $5^{\text {th }}$, is associated with the chaotic or turbulent dynamics of the flows, and so these larger modes become more significant with an increase in Re.

The POD enables a global picture of the flow decomposed on average patterns and coherent structures and associates to those modes an energy value. In addition to the flow spatial decomposition, the flow overall dynamics is also summarized in a single POD coefficients vector. The POD modes give a spatial description of the different flow structures, and since the decomposition is orthogonal in space, they enable to reconstruct the flow dynamics with the POD coefficients. The POD enables to compress the entire flow information by more than an order of magnitude. 
Table 1. Inlet velocity and time-step size for the various Re.

\begin{tabular}{ccc}
\hline $\boldsymbol{R} \boldsymbol{e}$ & $\boldsymbol{v}_{\boldsymbol{i n j}}(\mathrm{m} / \mathrm{s})$ & $\boldsymbol{\Delta t} \times \mathbf{1 0}^{-\mathbf{3}}(\mathrm{s})$ \\
\hline 250 & 3.33 & 6.25 \\
\hline 300 & 4.00 & 5.21 \\
\hline 400 & 5.33 & 3.91 \\
\hline 500 & 6.67 & 3.13 \\
\hline
\end{tabular}

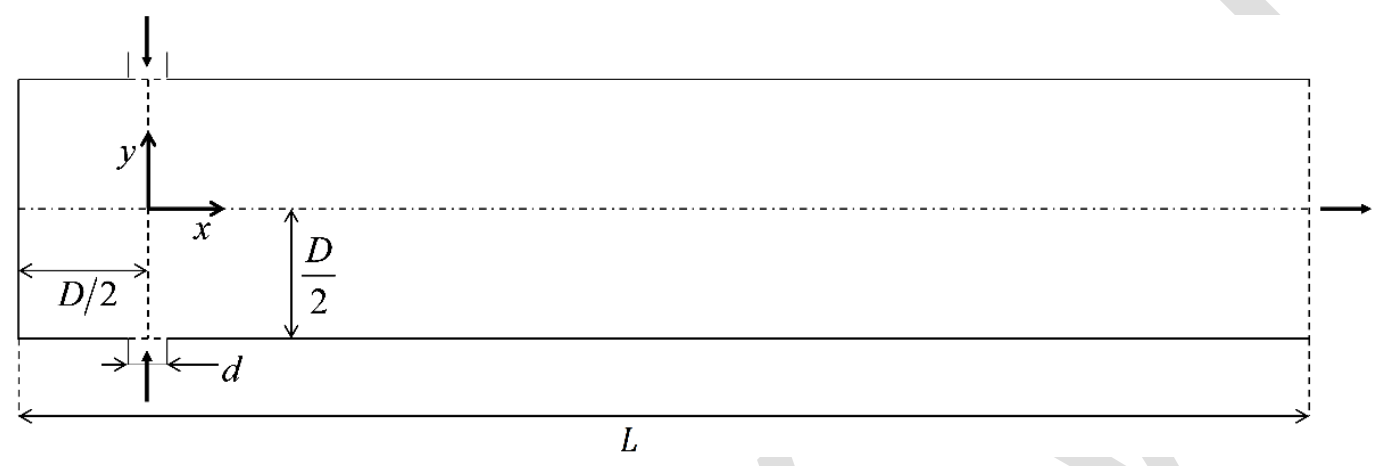

Figure 1. Geometry of the ClJ.

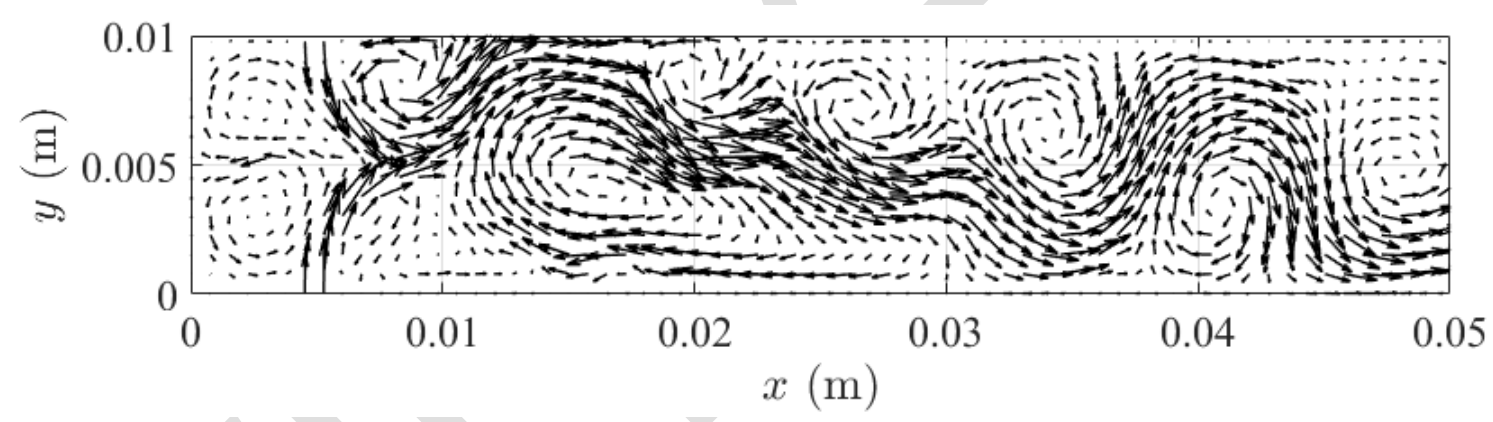

Figure 2. Velocity field at the end of 5 residence times. 


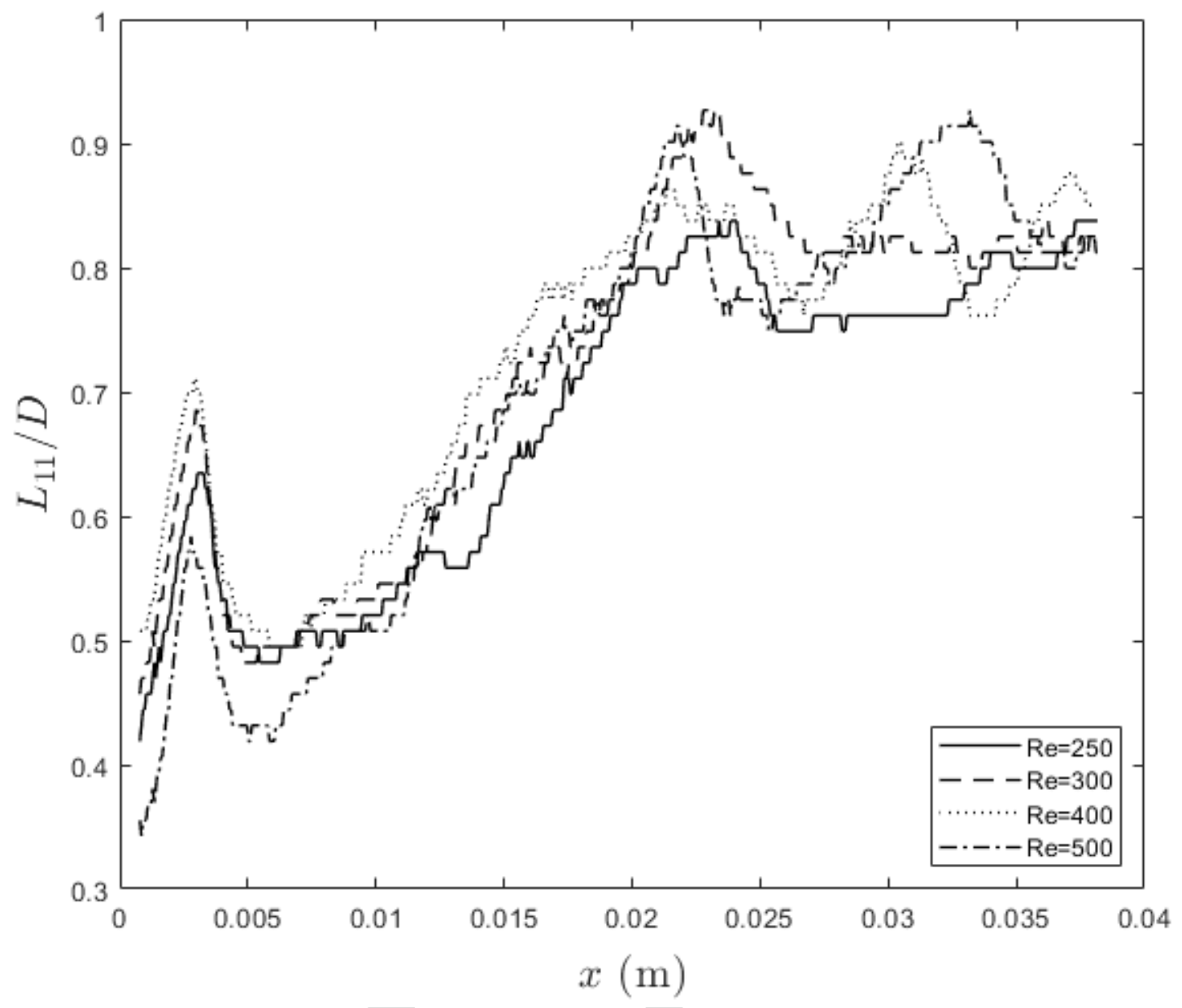

Figure 3. Turbulent lengthscales of the CIF at various Reynolds numbers: 250 (solid line), 300 (dashed line), 400 (dotted line) and 500 (dot-dashed line). 


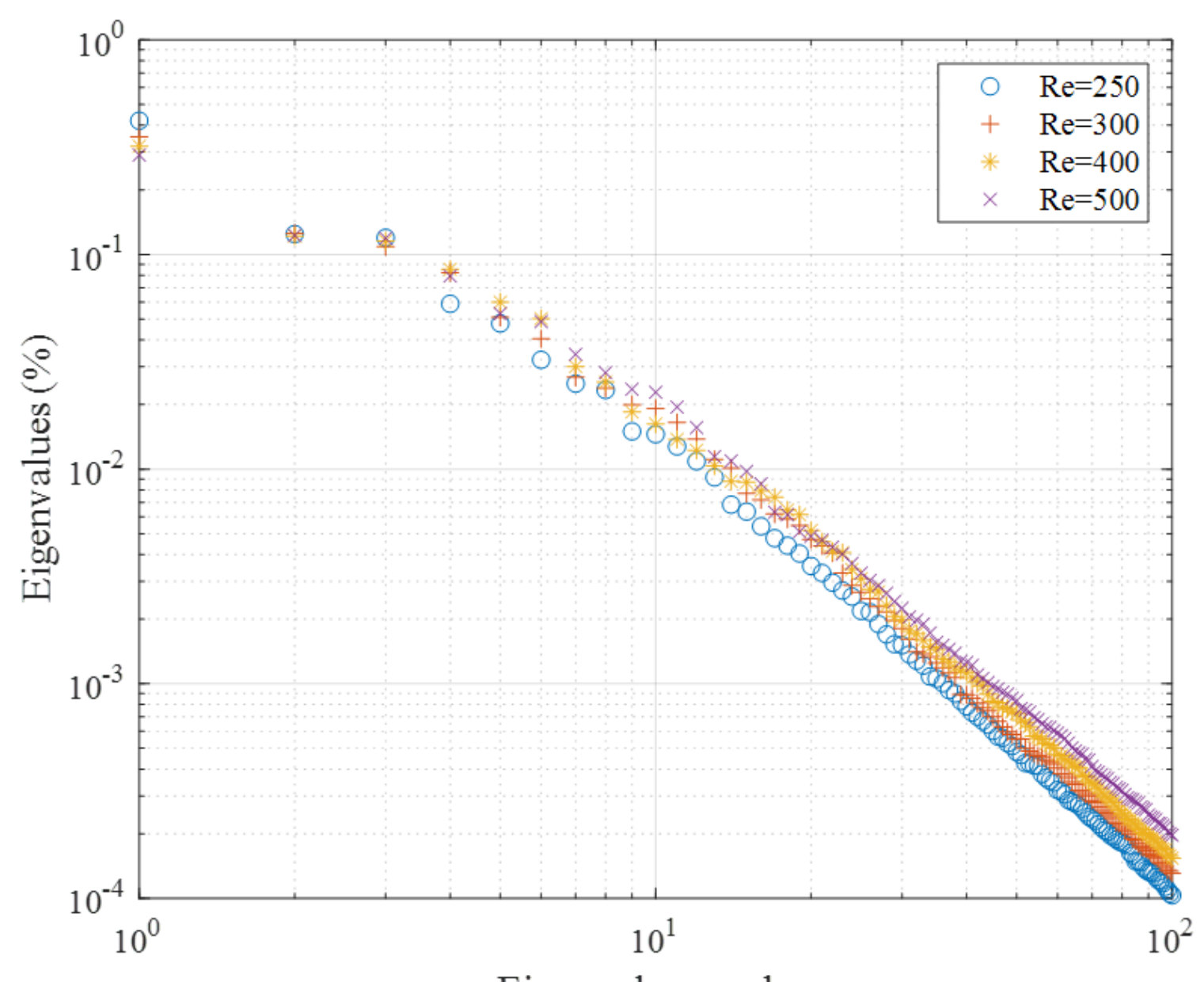

Eigenvalue number

Figure 4. Eigenvalue spectra for different Reynolds numbers: $R e=250$ (blue circle), $R e=300$ (red plus sign), $\operatorname{Re}=400$ (yellow asterisk) and $\mathrm{Re}=500$ (purple cross). 


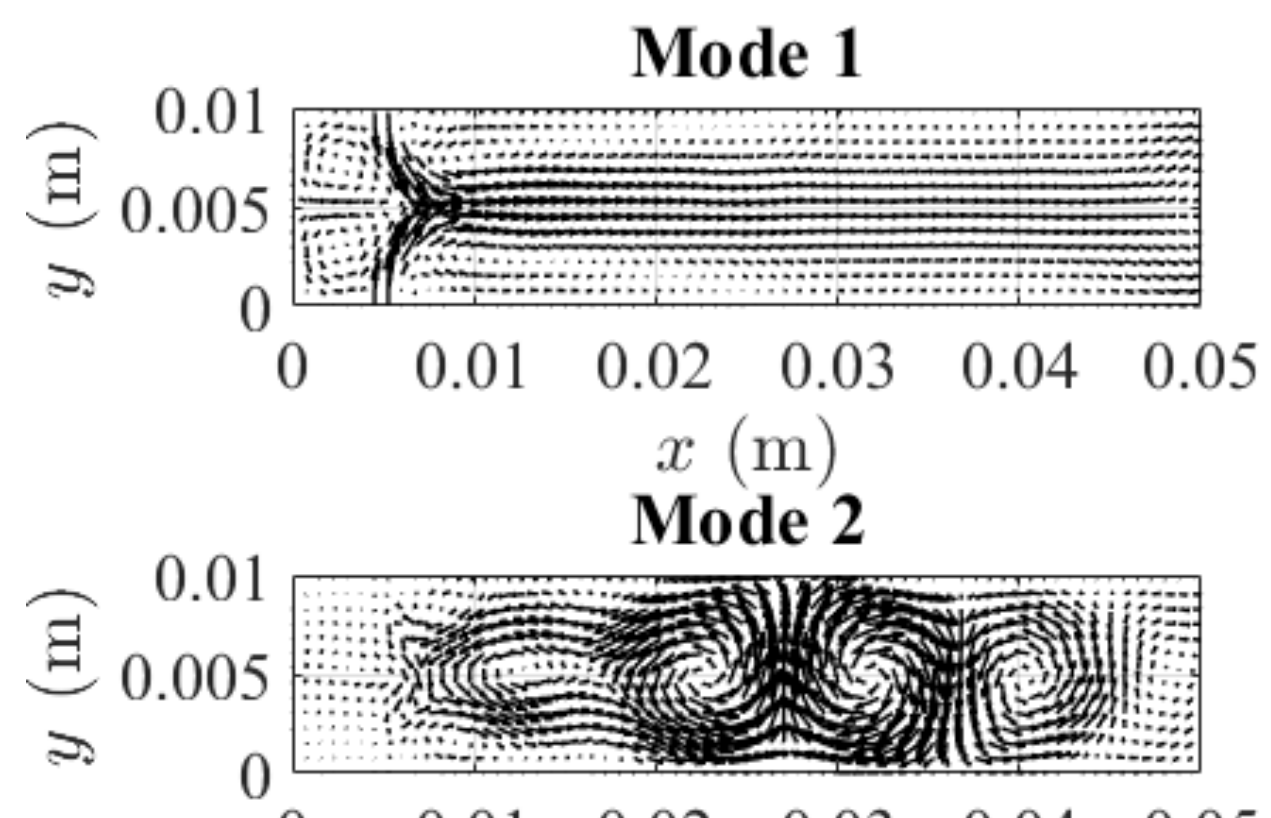

$\begin{array}{llllll}0 & 0.01 & 0.02 & 0.03 & 0.04 & 0.05\end{array}$

$x(\mathrm{~m})$

Mode 3
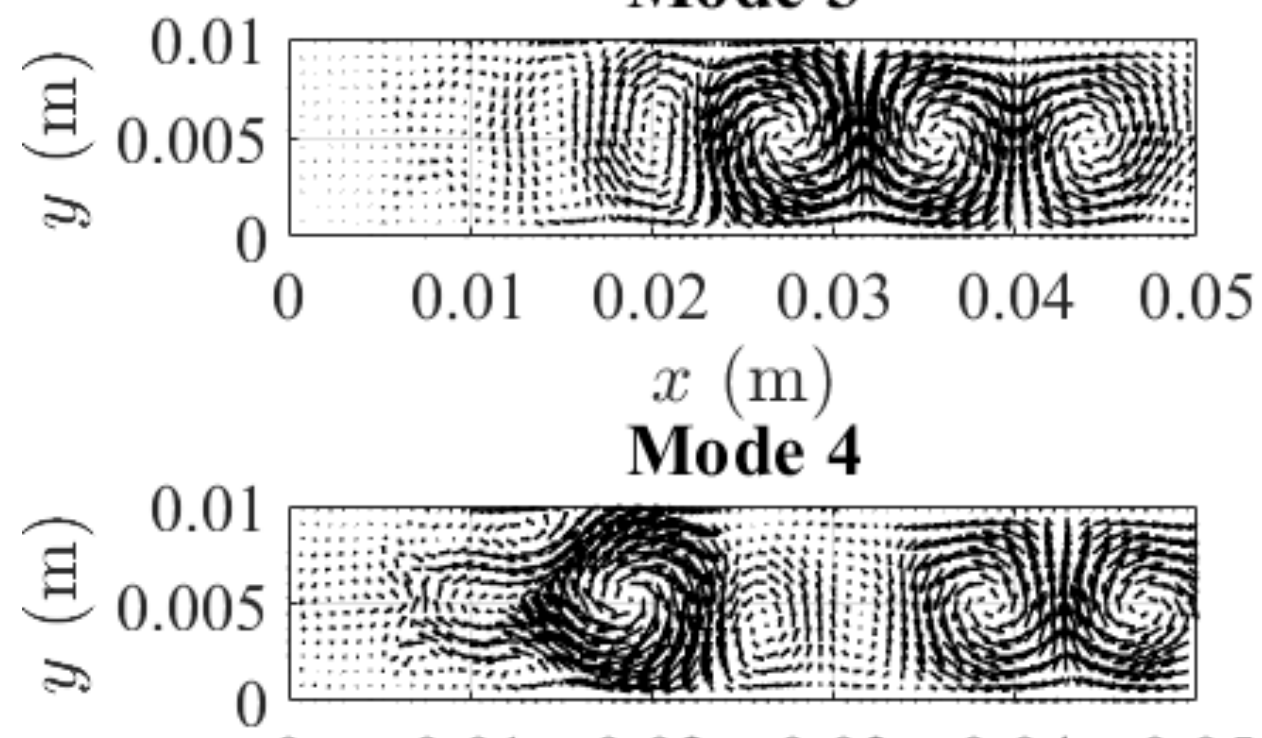

$\begin{array}{llllll}0 & 0.01 & 0.02 & 0.03 & 0.04 & 0.05\end{array}$

$x(\mathrm{~m})$

Mode 5

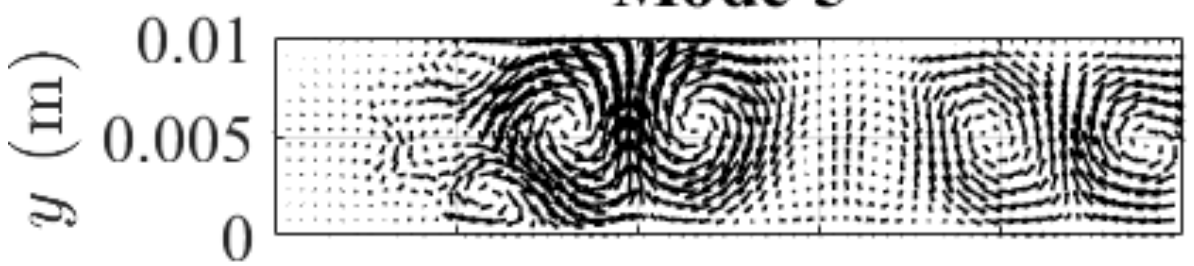

$\begin{array}{llllll}0 & 0.01 & 0.02 & 0.03 & 0.04 & 0.05\end{array}$

$$
x(\mathrm{~m})
$$

Figure 5. Vector fields for the first 5 modes' eigenvectors. From top to bottom: mode 1, mode 2, mode 3 , mode 4 and mode 5 . 

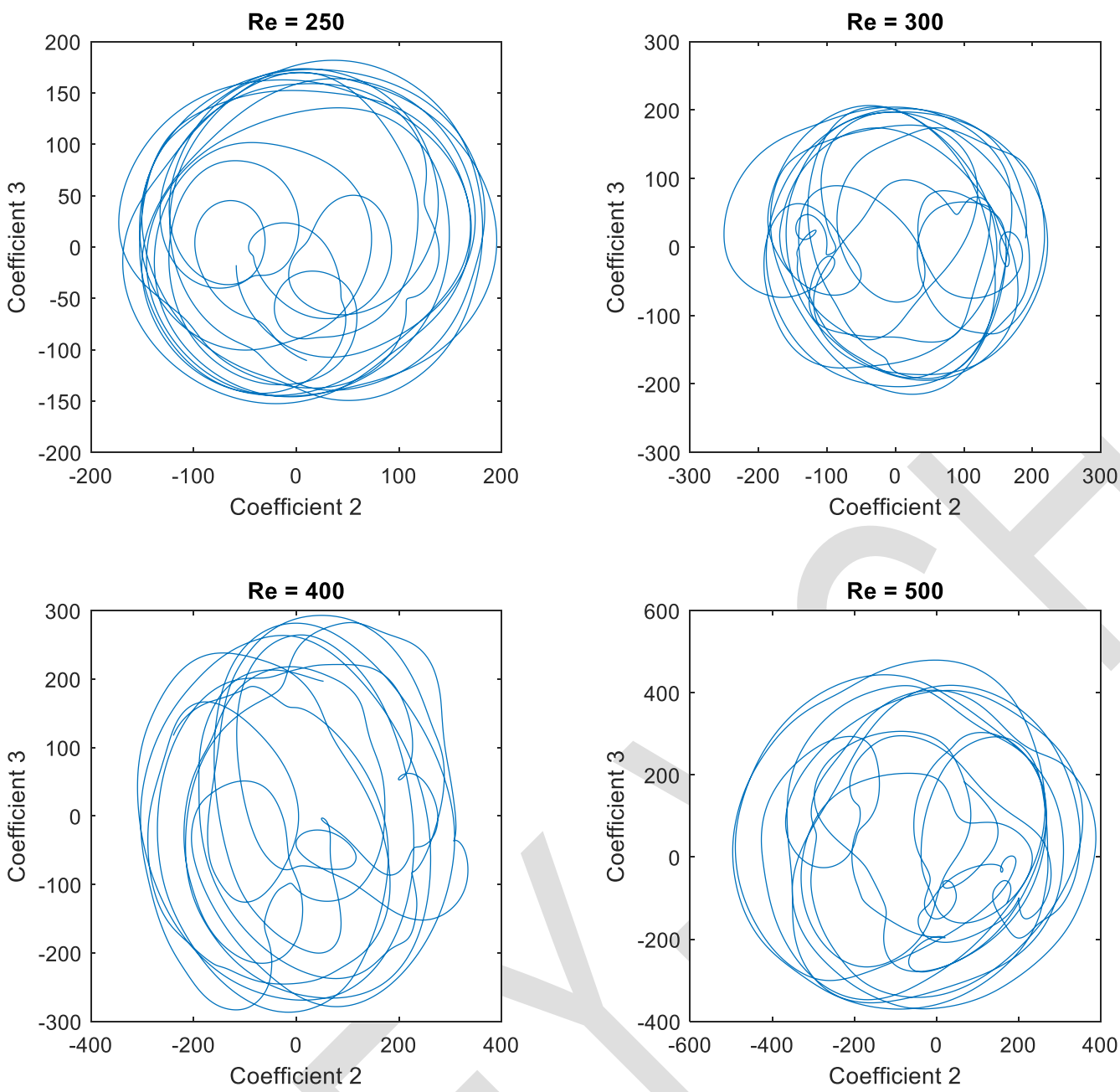

Figure 6. Relation between POD coefficients for modes 2 and 3 for Reynolds numbers of 250 , 300,400 and 500 


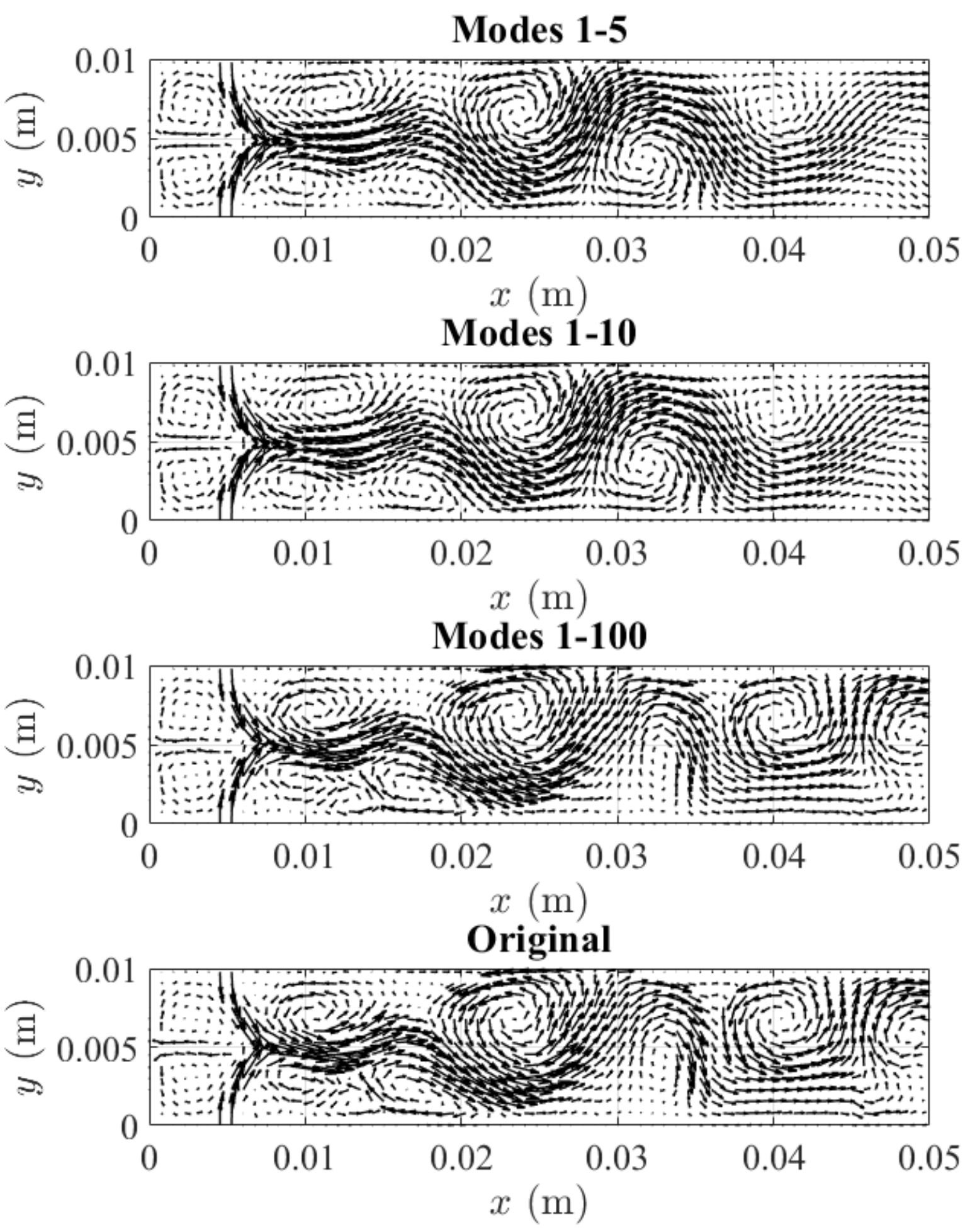

Figure 7. Reconstruction at $t=0$ (after 5 residence times), using 5 modes, 10 modes and 100 modes. The original field is shown for comparison. 


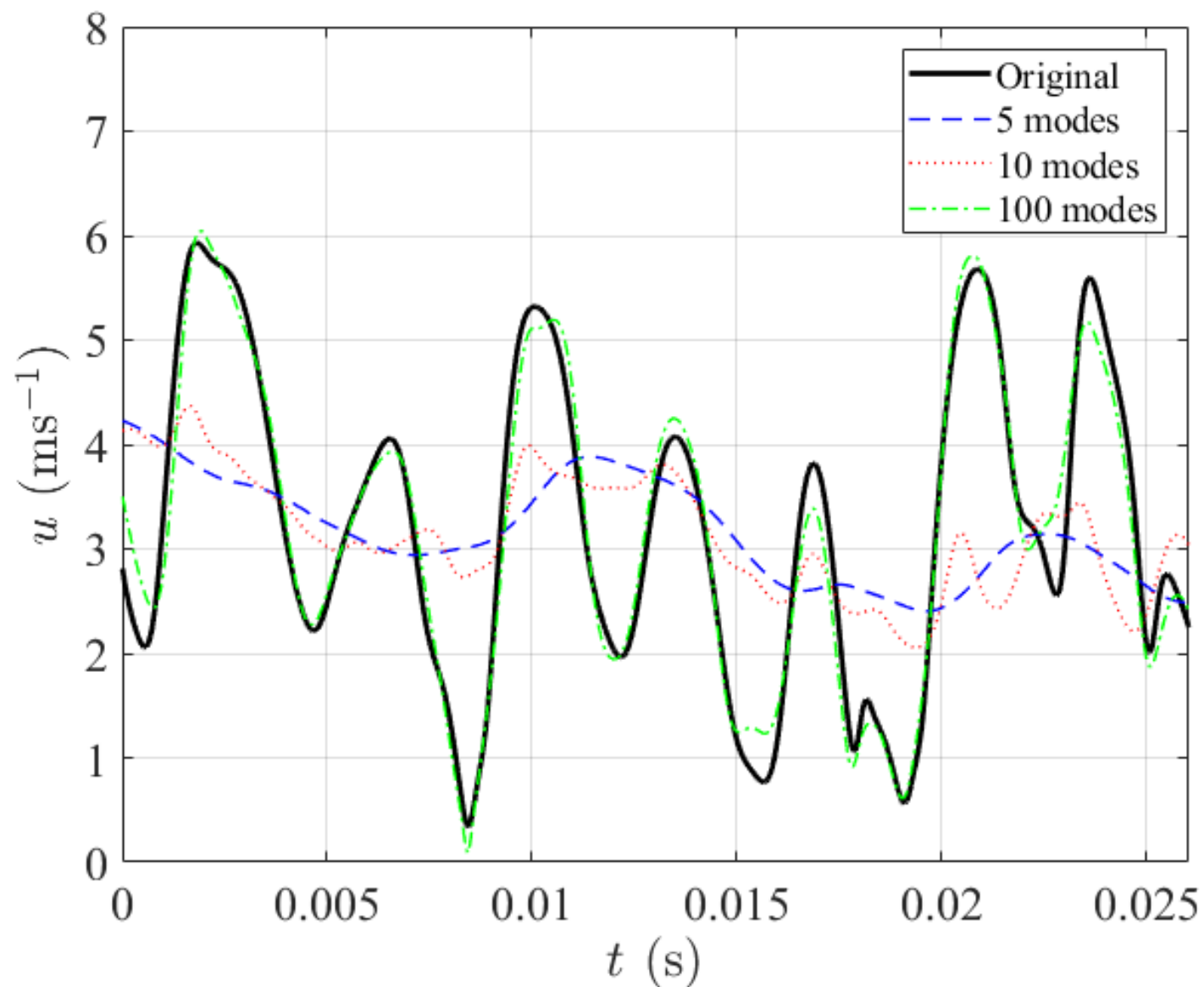

Figure 8. Velocity magnitude plot on the impingement point, using various velocity fields: original flow (solid black) and reconstructions with 5 modes (dashed blue), 10 modes (solid red) and 100 modes (solid green).

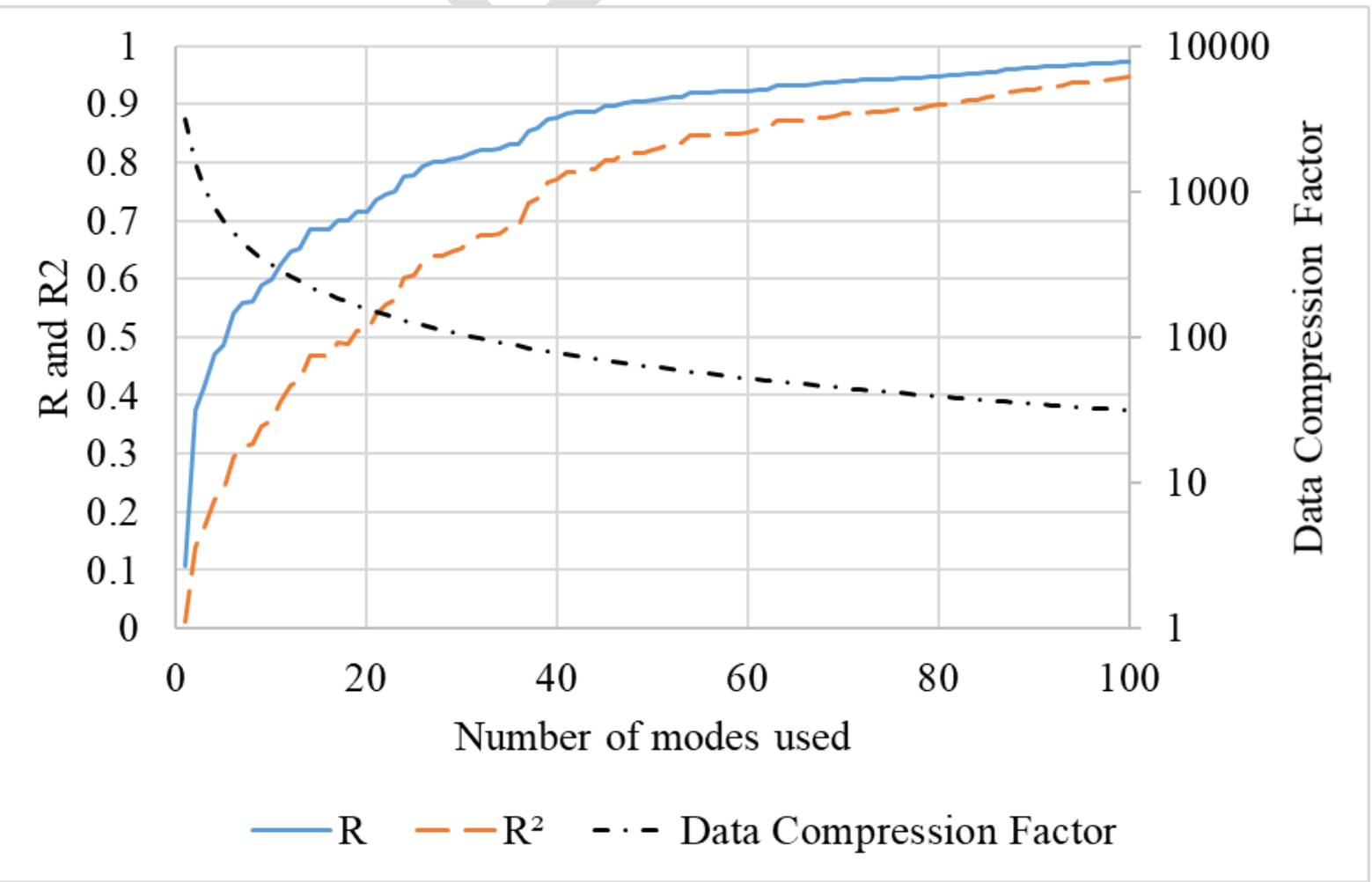

Figure 9. Correlation and Determination Coefficients versus number of modes used in the reconstruction (left axis); Data Compression Factor (right axis). 


\section{Acknowledgment}

This work is a result of project "AIProcMat@N2020 - Advanced Industrial Processes and Materials for a Sustainable Northern Region of Portugal 2020", with the reference NORTE-01-0145FEDER-000006, supported by Norte Portugal Regional Operational Programme (NORTE2020), under the Portugal 2020 Partnership Agreement, through the European Regional Development Fund (ERDF) and of Projects PTDC/QEQ-FTT/0041/2014, PTDC/EQU-EPQ/30445/2017, POCI01-0145-FEDER-030445, POCI-01-0145-FEDER-006984 - Associate Laboratory LSRE-LCM funded by ERDF through COMPETE2020 - Programa Operacional Competitividade e Internacionalização (POCI) - and by national funds (PIDDAC) through FCT/MCTES.

\section{Symbols used}

$\begin{array}{lll}a & & \text { POD coefficient } \\ d & {[\mathrm{~m}]} & \text { Inlet channels width } \\ D & {[\mathrm{~m}]} & \text { Chamber width } \\ L & {[\mathrm{~m}]} & \text { Chamber Length } \\ \boldsymbol{M} & & \text { Autocovariance matrix } \\ N & & \text { Number of points } \\ \mathcal{N} & & \text { Number of modes } \\ p & {[\mathrm{~Pa}]} & \text { Gauge pressure } \\ \mathbb{B} & & \text { Registered trademark } \\ r & & \text { Displacement bwtween two points } \\ R e & & \text { Reynolds number } \\ t & {[\mathrm{~s}]} & \text { Time } \\ \boldsymbol{u} & {[\mathrm{m} / \mathrm{s}] \text { (per component) }} & \text { Velocity vector } \\ \boldsymbol{U} & {[\mathrm{m} / \mathrm{s}] \text { (per component) }} & \text { Velocity field } \\ \boldsymbol{X} & {[\mathrm{m} / \mathrm{s}] \text { (per component) }} & \text { Complete history of velocity fields matrix }\end{array}$

Greek letters

$\begin{array}{cl}\boldsymbol{\alpha} & \\ \lambda & \\ \mu & {[\mathrm{Pa} \cdot \mathrm{s}]} \\ \rho & {\left[\mathrm{kg} \cdot \mathrm{m}^{-3}\right]} \\ \tau & {[\mathrm{s}]} \\ v_{i n j} & {[\mathrm{~m} / \mathrm{s}]}\end{array}$

\section{Eigenvector}

Eigenvalue

Kinematic Viscosity

Density

Residence Time

Average velocity at the inlets

Sub- and Superscripts$$
i
$$

inj

$j$

$k$

$n$

$x$
Row index

Injectors

Column index

Time-step

Number of mode

$x$ component 
$y$

Abbreviations

2D

CFD

$\mathrm{CIJ}$

POD $y$ component

Two dimensional

Computational Fluid Dynamics

Confined Impinging Jets

Proper Orthogonal Decomposition

\section{References}

[1] Y. C. Liang, H. P. Lee, S. P. Lim, W. Z. Lin, K. H. Lee, C. G. Wu, J. Sound Vib. 2002, 3, (252), 527-544, DOI: 10.1006/jsvi.2001.4041.

[2] P. Holmes, J. L. Lumley, G. Berkooz, "3. Proper orthogonal decomposition," Turbulence, Coherent Structures, Dynamical Systems and Symmetry, C. U. Press, Ed., 1996.

[3] D. D. Kosambi, J. Indian Math. Soc. 1943, (7), 76-88.

[4] M. Loève, Comptes Rendus Acad. Sci. Paris 1945, (220).

[5] K. Karhunen, Ann. Acad. Sci Fenn. 1946, Ser. A1, (34).

[6] S. S. Sapatnekar, IEEE J. Emerg. Sel. Top. Circuits Syst. 2011, 1, (1), 5-18, DOI: 10.1109/jetcas.2011.2138250.

[7] J. M. Ottino, The kinematics of mixing, Cambridge University Press, 1989.

[8] J. M. Ottino, S. C. Jana, V. S. Chakravarthy, Phys. Fluids 1994, 2, (6), 685-699, DOI: 10.1063/1.868308.

[9] A. Ducci, Z. Doulgerakis, M. Yianneskis, Ind. Eng. Chem. Res. 2008, 10, (47), 36643676, DOI: 10.1021/ie070905m.

[10] Z. Doulgerakis, M. Yianneskis, A. Ducci, AlChE J. 2011, 11, (57), 2941-2954, DOI: 10.1002/aic.12519.

[11] A. Liné, Chem. Eng. Res. Des. 2016, (108), 13-22, DOI: 10.1016/j.cherd.2015.10.023.

[12] A. Liné, J. C. Gabelle, J. Morchain, D. Anne-Archard, F. Augier, Chem. Eng. Res. Des. 2013, 11, (91), 2073-2083, DOI: 10.1016/j.cherd.2013.05.002.

[13] W. H. Weheliye, N. Cagney, G. Rodriguez, M. Micheletti, A. Ducci, Phys. Fluids 2018, 3, (30), 033603, DOI: 10.1063/1.5016305.

[14] R. J. Santos, A. M. Teixeira, M. R. P. F. N. Costa, J. C. B. Lopes, Int. Polym. Process. 2002, 4, (17), 387-394.

[15] R. J. Santos, A. M. Teixeira, J. C. B. Lopes, Chem. Eng. Sci. 2005, 8-9, (60), 23812398, DOI: Doi 10.1016/J.Ces.2004.11.050.

[16] R. J. Santos, A. M. Teixeira, E. Erkoç, M. A. Sultan, A. Karpinska, M. M. Dias, J. C. B. Lopes, Int. J. Chem. React. Eng. 2010, (8), A32.

[17] R. J. Santos, E. Erkoç, M. M. Dias, J. C. B. Lopes, AlChE J. 2009, 6, (55), 1338-1351, DOI: 10.1002/aic.11771.

[18] C. P. Fonte, R. J. Santos, M. M. Dias, J. C. B. Lopes, Int. J. Chem. React. Eng. 2011, A114, (9), DOI: 10.2202/1542-6580.2564.

[19] C. A. P. da Fonte, Mixing Studies with Impinging Jets, Universidade do Porto, Faculdade de Engenharia , 2012, Vol. PhD.

[20] N. D. Gonçalves, H. M. Salvador, C. P. Fonte, M. M. Dias, J. C. B. Lopes, R. J. Santos, AIChE J. 2017, DOI: 10.1002/aic.15566. 
[21] R. J. Santos, M. A. Sultan, Chem. Eng. Technol. 2013, 6, (36), 937-949, DOI: Doi 10.1002/Ceat.201200678.

[22] J. Matos, M. S. C. A. Brito, M. M. Dias, J. C. B. Lopes, R. J. Santos, Chem. Eng. Sci. 2018, (192), 199-210, DOI: 10.1016/J.CES.2018.07.035.

[23] S. B. Pope, Turbulent flows, Cambridge Univ. Press, Cambridge [u.a.], 2001.

[24] A. Liné, Chem. Eng. Res. Des. 2016, (108), 13-22, DOI: 10.1016/j.cherd.2015.10.023.

[25] L. Sirovich, Phys. D Nonlinear Phenom. 1989, 1, (37), 126-145, DOI: https://doi.org/10.1016/0167-2789(89)90123-1.

\section{Table and Figure captions}

Table 1. Inlet velocity and time-step size for the various Re.

Figure 1. Geometry of the ClJ.

Figure 2. Velocity field at the end of 5 residence times.

Figure 3. Turbulent lengthscales of the CIF at various Reynolds numbers: 250 (solid line), 300 (dashed line), 400 (dotted line) and 500 (dot-dashed line).

Figure 4. Eigenvalue spectra for different Reynolds numbers: $R e=250$ (blue circle), $R e=300$ (red plus sign), $\operatorname{Re}=400$ (yellow asterisk) and $\mathrm{Re}=500$ (purple cross).

Figure 5. Vector fields for the first 5 modes' eigenvectors. From top to bottom: mode 1, mode 2, mode 3 , mode 4 and mode 5 .

Figure 6. Relation between POD coefficients for modes 2 and 3 for Reynolds numbers of 250, 300,400 and 500

Figure 7. Reconstruction at $t=0$ (after 5 residence times), using 5 modes, 10 modes and 100 modes. The original field is shown for comparison.

Figure 8. Velocity magnitude plot on the impingement point, using various velocity fields: original flow (solid black) and reconstructions with 5 modes (dashed blue), 10 modes (solid red) and 100 modes (solid green).

Figure 9. Correlation and Determination Coefficients versus number of modes used in the reconstruction (left axis); Data Compression Factor (right axis). 